\title{
MBE growth Monitoring of a single GaAs nanowire by in-situ X-ray nano-diffraction
}

\author{
S. M Mostafavi Kashani ${ }^{1}$, J. Vogel ${ }^{1}$, A. Davtyan' ${ }^{1}$, D. Bahrami ${ }^{1}$, L. Feigl ${ }^{3}$, J. Jakob ${ }^{2,3}$, P. Schroth ${ }^{1,2,3}$, T. Baumbach $^{2,3}$, \\ U. Pietsch ${ }^{1}$
}

\author{
${ }^{1}$ University of Siegen, Solid State Physics, Siegen, Germany \\ ${ }_{2}^{2}$ Institute for Photon Science and Synchrotron Radiation (IPS), Karlsruhe Institute of Technology (KIT), Karlsruhe, Germany; \\ ${ }^{3}$ Laboratory for Application of Synchrotron Radiation (LAS), Karlsruhe Institute of Technology (KIT), Karlsruhe, Germany
}

pietsch@physik.uni-siegen.de

For the first time the growth of a single GaAs nanowire (NW) was monitored by in-situ time resolved X-ray nano-diffraction (nXRD) using focused synchrotron radiation at beamline P09 of PETRA III storage ring (Hamburg) and a portable MBE chamber [1]. A particular position for nucleation and growth was selected within an array of holes with 5 micron pitch prepared on a lithography-free pre-patterned $\mathrm{Si}(111)$ substrate covered by $16 \mathrm{~nm}$ thick oxide [2]. Exploiting a photon flux of $4 \cdot 10^{9} \mathrm{Photons}^{-1} \mathrm{~s}^{-1}$ focused in a microbeam of $2 \times 6$ micon $^{2}$ and probing the GaAs 111 Bragg reflection the first NW signal of above the background did appear about 24 minutes after opening the Ga and As shutters, corresponding to a NW length and diameter of about $45 \mathrm{~nm}$ and $28 \mathrm{~nm}$, respectively. The time evolution of the NW signal could be monitored for 55 minutes up to the final length and diameter of about $2000 \mathrm{~nm}$ and $45 \mathrm{~nm}$, respectively. Both parameters and the NW orientation with respect to the substrate normal were evaluated from the peak intensity and peak shape and position after background correction and separation of the NW signal from that of a parasitic island growing within the same probing volume. The final NW dimensions extracted from XRD analysis are in good agreement with ex-situ SEM data taken from the same NW after growth. In the experiment reported the observed time evolution of NW growth follows two subsequent stages: 1) dominant axial growth accompanied by unstable axial orientation of the NW followed by 2) increase of radial growth at stable axial orientation. Although successful proof of principle, quantitatively the experiment suffered from tiny fluctuations of the spatial position of the micro-beam during the entire growth cycle and/or limitations in the accuracy of angle settings.

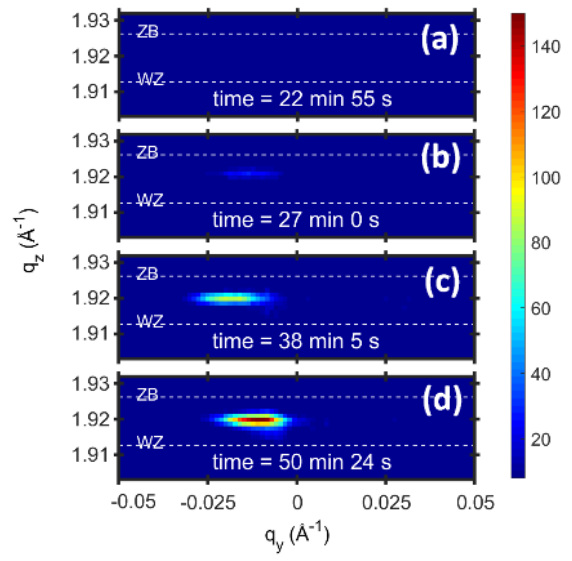

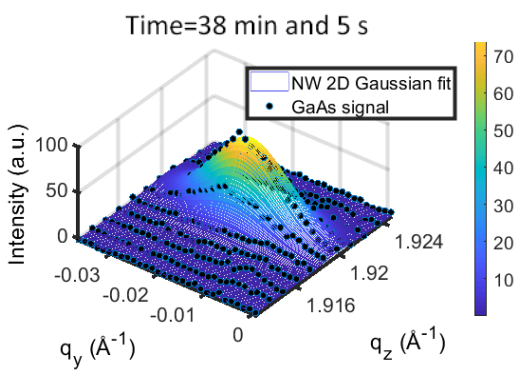

(d)

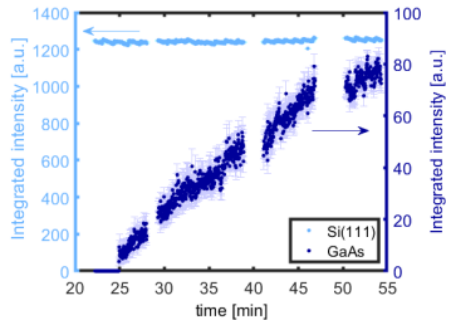

(e)

Figure 1: Panels (a - d) show four representative detector frames transformed into the reciprocal space after background removal. 2D intensity distribution at about time $=\mathbf{2 2} \mathbf{m i n}$ was accounted as the background and subtracted from all detectors. The color bar at the right is linearly scaled and belongs to all detector frames. (d) Two-dimensional Gaussian fitting of the GaAs signal at the $q \mathrm{y}-\mathrm{q} \mathrm{z}$ intensity distribution at the detector frame acquired at time $=38 \mathrm{~min}$ and $5 \mathrm{~s}$. (e) The integrated intensity of the NW signal during growth: $1.4 \mathrm{~s}$ effective time per frame. Here, light blue is the Si signal (left axis); dark blue is the GaAs NW signal (right axis). On the gaps, RSM and/or RS is performed.

[1] Schroth, P., Jakob, J.,Feigl, L., Mostafavi Kashani,S. M., Vogel, J., Strempfer, J., Keller, T.F., Pietsch, U. and Baumbach, T. (2018) Nano Lett. 18, pp. 101-108,

[2] Bahrami, D., Mostafavi Kashani, S.M., AlHassan, A., Davtyan, A., and U Pietsch U. (2020) Nanotechnology 31, pp. 185302

\section{Keywords: GaAs nanowires, nano-diffraction, in-situ MBE growth}

Acknowledgement: This work was supported by BMBF Verbundforschung under 05K16PSA. 